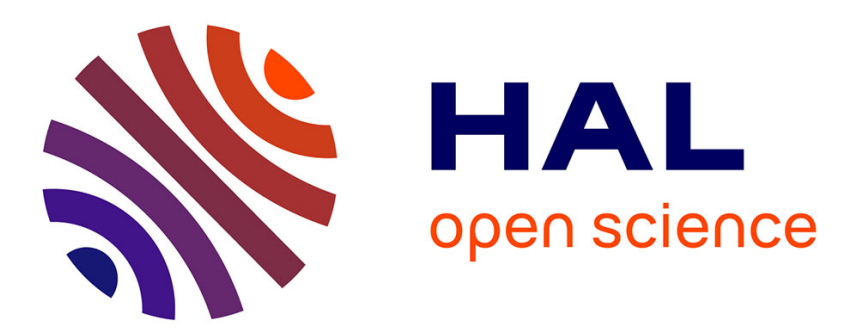

\title{
Estimation of SEA coupling loss factors using a dual formulation and FEM modal information, part I: theory \\ Laurent Maxit, Jean-Louis Guyader
}

\section{To cite this version:}

Laurent Maxit, Jean-Louis Guyader. Estimation of SEA coupling loss factors using a dual formulation and FEM modal information, part I: theory. Journal of Sound and Vibration, 2001, 239 (5), pp.907930. 10.1006/jsvi.2000.3192 . hal-01162185v1

\section{HAL Id: hal-01162185 \\ https://hal.science/hal-01162185v1}

Submitted on 9 Jun 2015 (v1), last revised 11 Sep 2020 (v2)

HAL is a multi-disciplinary open access archive for the deposit and dissemination of scientific research documents, whether they are published or not. The documents may come from teaching and research institutions in France or abroad, or from public or private research centers.
L'archive ouverte pluridisciplinaire HAL, est destinée au dépôt et à la diffusion de documents scientifiques de niveau recherche, publiés ou non, émanant des établissements d'enseignement et de recherche français ou étrangers, des laboratoires publics ou privés. 


\title{
CALCULATION OF SEA COUPLING LOSS FACTORS USING THE DUAL FORMULATION AND FEM MODAL INFORMATION PART I: THEORY
}

\author{
Authors: $\quad$ Laurent MAXIT and Jean-Louis GUYADER \\ Address: $\quad$ INSA de Lyon (National Institute of Applied Sciences) \\ L.V.A. (Vibrations - Acoustic Laboratory) \\ Bat. 303 \\ 20, avenue Albert Einstein \\ 69621 Villeurbanne Cedex \\ France
}

Phone: $\quad+33472438080$

+33472438712 (fax)

Email: $\quad$ maxit@lva.insa-lyon.fr, guyader@lva.insa-lyon.fr

Headline: SEA COUPLINGS LOSS FACTORS

Total number of pages: 41

Total number of figures: 6

$\underline{\text { Total number of footnotes: } 1}$ 


\section{Summary:}

The theoretical approach presented in this paper allows us to calculate SEA Coupling Loss Factors for subsystems modelled with FEM. It is then possible to take into account complicated substructure that can be encountered in practical industrial application. The technique relies on the basic SEA relation for coupled oscillators and the use of Dual Modal Formulation to describe vibration of coupled subsystems. With this approach, the boundary conditions of uncoupled subsystems are clearly defined and as assumed in SEA no modal coupling exists in a subsystem. Modes of two different subsystems are coupled together by gyroscopic elements and the coupling strength is related to eigenfrequencies of the uncoupled subsystems and mode shapes through the interaction modal works. A general expression of CLF has been obtained, it permits to determine CLF only from the knowledge of the modes of the uncoupled subsystems and the modal damping. Finite Element Model can be used to calculate the modal information in the case of complex substructures. It is possible to treat the case of heterogeneous subsystems having three dimensional vibration motions without difficulty. Contrary to classical approach based on SEA inverse matrix and numerical experiments, that necessitates calculations of subsystem energies for the coupled structures for a lot of excitation points, this technique calculates CLF directly from the governing equations, without solving them. In a companion paper, the present approach is applied about simple example to illustrate it and to present validations. 


\section{INTRODUCTION}

Statistical Energy Analysis (S.E.A.) permits to predict vibro-acoustic behaviour of complex structures in mid and high frequency range. The method relates the power flow exchanged by two coupled subsystems to total subsystem energies by the so-called Coupling Loss Factor (CLF). Writing finally the power balance for stationary motion in each subsystem produces a linear equation system where the unknowns are total energies of subsystems. Then, the difficulty in applying SEA is not due to solving complicated equations, but to the evaluation of Coupling Loss Factors.

Several techniques have been developed to determine CLF. The travelling wave approach is the most popular to obtain theoretical expression in simple cases of coupled beams, plates, and shells (see[1], Chapter 10). Based on the evaluation of the wave transmission coefficient, this approach is very easy to use. However, it can lead to mistake for system having a low modal overlap (see [2]). CLF can be only calculated for academic substructures that limits the application in the cases of manufacturing structures. Different experimental approaches have been elaborated to evaluate CLF by measurement:[3] using measured point mobility; [4] with the concept of energetic mobility; [5] from measured impulse responses in the coupled system; [6], [7], [8] based about inversion of the SEA equation. This last one, called the power injection method is the most popular. The difficulty of the method relies on the number of transfer functions that are necessary to measure, also that the evaluation of the spatial averaged energy from some measurement points in the case of heterogeneous subsystems. These experimental techniques are very useful in an industrial context but they necessitate to have the mechanical structure, then, it is not a predictive method. To solve this problem, the possibility to use Finite Elements Method to calculate numerically the response of two coupled subsystems and then identify CLF has been used [9], [10], [11]. This type of approach is predictive, and has been used to study the validity of some SEA assumptions. The difficulties of 
this approach are similar to experimental approach for heterogeneous substructures. The limit of application are, of course, the frequency range link to use FEM, and the difficulty to give different damping loss factor to the various subsystems because global modes are used. The approach presented in reference [12] differs from the previous method by the use of the numerical Green functions of the un-coupled subsystems and a receptance based approach. Then it is possible to take into account different damping for various subsystems and to increase, in some cases, the frequency range considered because the FEM calculation are made about individual uncoupled subsystem. However, it is necessary to include sufficient uncoupled modes to constitute the Green function that can be a frequency limitation.

The approach presented in this paper allows us to calculate CLF directly from subsystem modal equations. The method uses modal definition of CLF which are established in the basic SEA formulation (see [1] Chapter $3,[13],[14]$ ) and remembered in section 2. The expression of the power flow exchanged by two oscillators coupled by gyroscopic element will be used to calculate the Coupling Loss Factor by summation of the different Intermodal Coupling Factors. One chose to consider deterministic structure although that it is easy possible to consider ensemble averaged over a population of structure. In section 3, one will see the major theoretical contribution of this paper. The purpose of this section is to propose one general approach allowing one to obtain modal equations of motion which can be represented as set of coupled oscillators (modes) by gyroscopic elements. These modal equations will permit to identify modal coupling coefficients necessary to calculate Intermodal Coupling Factors and thus, Coupling Loss Factors. The formulation will be presented in the general case of the two coupled continuous three-dimensional elasto-dynamic systems. This approach is based on two subsystem modes definitions, and on the use of the Dual Modal Formulation (D.M.F). It is analogous to the approach used to describe the mechanical structure - cavity coupling [15], [16], [17]. Therefore one presents here a generalisation like suggested in reference [18]. 
In the last section, D.M.F. is applied to discretized system to determine the modal coupling coefficients. Then, in the cases of complex subsystems, FEM can be used to calculate the modes of each uncoupled subsystem and to deduce Coupling Loss Factor.

\section{CLF EXPRESSION DEDUCED FROM MODAL SEA FORMULATION}

\subsection{POWER FLOW EXCHANGED BY TWO OSCILLATORS COUPLED BY GYROSCOPIC ELEMENT}

\section{FIGURE 1}

Two oscillators coupled via a gyroscopic element (see figure 1) are considered. $M_{1}, M_{2}$ are the masses, and $K_{1}, K_{2}$ are the stiffness of the oscillators. Then, the natural angular frequencies of each uncoupled oscillator are: $\omega_{1}=\sqrt{K_{1} \cdot M_{1}^{-1}}$ for oscillator 1 and $\omega_{2}=\sqrt{K_{2} \cdot M_{2}^{-1}}$ for oscillator 2 .

Each oscillator is damped by viscous absorber of damping coefficient: $\Delta_{1}$ for oscillator 1 and $\Delta_{2}$ for oscillator 2. The coupling forces transmitted through the coupling of constant $\mathrm{G}_{\mathrm{c}}$ are proportional to the mass velocities, $\dot{y}_{1}$ and $\dot{y}_{2}$. It is assumed that when the velocity of oscillator 1 is positive, the force applied on oscillator 2 is negative and when the velocity of oscillator 2 is positive, the force applied on oscillator 1 is positive. Then, the equations of motion for the two coupled oscillators excited by external forces $F_{1}$ and $F_{2}$ are yielded by:

$$
\left\{\begin{array}{l}
\ddot{y}_{1}+\Delta_{1} \cdot \dot{y}_{1}+\omega_{1}^{2} \cdot y_{1}-\sqrt{M_{1}^{-1} \cdot M_{2}} \cdot \gamma \cdot \dot{y}_{2}=F_{1}, \\
\ddot{y}_{2}+\Delta_{2} \cdot \dot{y}_{2}+\omega_{2}^{2} \cdot y_{2}+\sqrt{M_{1} \cdot M_{2}^{-1}} \cdot \gamma \cdot \dot{y}_{1}=F_{2},
\end{array}\right.
$$

where the gyroscopic modal coupling coefficient, $\gamma$, is:

$$
\gamma=\frac{G_{C}}{\sqrt{M_{1} \cdot M_{2}}}
$$


Now, one assumes that external excitations are independent (uncorrelated), stationary and to have PSD constant (white noise). It has been demonstrated in this case [1] that the time averaged power flow from oscillator 1 to oscillator $2, \Pi_{12}$, is proportional to the difference of the time averaged total energies of the oscillators $\left(E_{1}-E_{2}\right)$ :

$$
\begin{gathered}
\Pi_{12}=\beta \cdot\left(E_{1}-E_{2}\right) \\
\text { where the coefficient } \beta \text { are expressed by: } \\
\beta=\frac{\gamma^{2}\left(\Delta_{1} \cdot \omega_{2}^{2}+\Delta_{2} \cdot \omega_{1}^{2}\right)}{\left(\omega_{1}^{2}-\omega_{2}^{2}\right)^{2}+\left(\Delta_{1}+\Delta_{2}\right)\left(\Delta_{1} \cdot \omega_{2}^{2}+\Delta_{2} \cdot \omega_{1}^{2}\right)} .
\end{gathered}
$$

One notices that the coefficient $\beta$ depends on the natural angular frequencies of the uncoupled oscillators, the damping constants and the coupling coefficient $\gamma$.

\subsection{FROM TWO COUPLED OSCILLATORS TO TWO COUPLED SUBSYSTEMS}

Now consider two coupled mechanical subsystems. The interaction between these subsystems may correspondingly be studied by investigating the interaction between two sets of resonant modes in the considered frequency band. This assertion which is expressed with little attention in the literature will be the subject of a detailed study in section 3 . One will show that for the good choice of modes and using dual modal formulation, the modal equations of motions have the form:

$$
\left\{\begin{array}{c}
\ddot{c}_{p}^{1}(t)+\Delta_{p}^{1} \cdot \dot{c}_{p}^{1}(t)+\left(\omega_{p}^{1}\right)^{2} \cdot c_{p}^{1}(t)=\frac{F_{p}^{1}}{\left(\omega_{p}^{1}\right)^{2} \cdot M_{p}^{1}}+\sum_{r=1}^{N_{2}}\left(\sqrt{\frac{M_{r}^{2}}{\left(\omega_{p}^{1}\right)^{2} \cdot M_{p}^{1}}} \cdot \gamma_{p r}^{12} \cdot \dot{a}_{r}^{2}(t)\right) \\
\ddot{a}_{q}^{2}(t)+\Delta_{q}^{2} \cdot \dot{a}_{q}^{2}(t)+\left(\omega_{q}^{2}\right)^{2} \cdot a_{q}^{2}(t)=\frac{F_{q}^{2}}{M_{q}^{2}}-\sum_{m=1}^{N_{1}}\left(\sqrt{\frac{\left(\omega_{m}^{1}\right)^{2} \cdot M_{m}^{1}}{M_{q}^{2}}} \cdot \gamma_{m q}^{12} \cdot \dot{c}_{m}^{1}(t)\right) \\
\forall p \in\left[1, N_{1}\right], \quad \forall q \in\left[1, N_{2}\right]
\end{array}\right.
$$


where: $c_{p}^{1}(t), M_{p}^{1}, \omega_{p}^{1}$ (resp. $\left.a_{q}^{2}(t), M_{q}^{2}, \omega_{q}^{2}\right)$ are modal amplitudes, generalised masses, and natural frequencies of subsystem 1 (resp. subsystem 2),

$F_{p}^{1}$ (resp. $F_{q}^{2}$ ) are generalised 'forces' applied on subsystem 1 (resp. subsystem 2),

$\gamma_{p q}^{12}$ are the modal coupling coefficients between couple of modes.

One considers that in the frequency band, there are $\mathrm{N}_{1}$ resonant modes for subsystem 1 and $\mathrm{N}_{2}$ resonant modes for subsystem 2. One assumes, and the choice of modes of section 3 will go in this direction, that these resonant modes can approximately represent the dynamic behaviour of the coupled subsystems in the frequency band considered.

Then, equations (5) can be schematically represented as figure 2: One mode of one subsystem is not coupled with modes of the same subsystem but is coupled by gyroscopic elements with the modes of the other subsystem.

\section{FIGURE 2}

Isolating, in the equations system, the coupling between the mode 'p' of subsystem 1 and the mode 'q' of subsystem 2, one can write:

$$
\left\{\begin{array}{l}
\ddot{c}_{p}^{1}(t)+\Delta_{p}^{1} \cdot \dot{c}_{p}^{1}(t)+\left(\omega_{p}^{1}\right)^{2} \cdot c_{p}^{1}(t)-\sqrt{\frac{M_{q}^{2}}{M_{p}^{1} \cdot\left(\omega_{p}^{1}\right)^{2}}} \cdot \gamma_{p q}^{12} \cdot \dot{a}_{q}^{2}(t)=\frac{L_{1 p q}}{M_{p}^{1} \cdot\left(\omega_{p}^{1}\right)^{2}}, \\
\ddot{a}_{q}^{2}(t)+\Delta_{q}^{2} \cdot \dot{a}_{q}^{2}(t)+\left(\omega_{q}^{2}\right)^{2} \cdot a_{q}^{2}(t)+\sqrt{\frac{M_{p}^{1} \cdot\left(\omega_{p}^{1}\right)^{2}}{M_{q}^{2}}} \cdot \gamma_{p q}^{12} \cdot \dot{c}_{p}^{1}(t)=\frac{L_{2 p q}}{M_{q}^{2}}
\end{array}\right.
$$

where the terms $L_{1 p q}$ and $L_{2 p q}$ contain the generalised forces and the interactions forces with all the others modes.

Then, by analogy with the equations of motions of two coupled oscillators (1), the power flow, $\Pi_{p q}^{12}$, exchanged by these two modes can be deduced: 


$$
\Pi_{p q}^{12}=\beta_{p q}^{12}\left(E_{p}^{1}-E_{q}^{2}\right)
$$

where: $\quad E_{p}^{1}, E_{q}^{2}$ are the modal energies of mode 'p' of subsystem 1 and mode 'q' of subsystem 2 ,

$\beta_{p q}^{12}$, called the Intermodal Coupling Factor (I.C.F.), can be determined by analogy with (4) in function of the natural angular frequencies $\omega_{p}^{1}, \omega_{q}^{2}$, the modal damping bandwidth $\Delta_{p}^{1}, \Delta_{q}^{2}$ and the modal coupling coefficient $\gamma_{p q}^{12}$ :

$$
\beta_{p q}^{12}=\frac{\left(\gamma_{p q}^{12}\right)^{2} \cdot\left(\Delta_{p}^{1} \cdot\left(\omega_{q}^{2}\right)^{2}+\Delta_{q}^{2} \cdot\left(\omega_{p}^{1}\right)^{2}\right)}{\left(\left(\omega_{p}^{1}\right)^{2}-\left(\omega_{q}^{2}\right)^{2}\right)^{2}+\left(\Delta_{p}^{1}+\Delta_{q}^{2}\right) \cdot\left(\Delta_{p}^{1} \cdot\left(\omega_{q}^{2}\right)^{2}+\Delta_{q}^{2} \cdot\left(\omega_{p}^{1}\right)^{2}\right)} .
$$

The application of relation (3) for the coupling of these two modes implies that one supposes that the forces $L_{1 p q}$ and $L_{2 p q}$ are uncorrelated, stationary, and have relatively flat spectra in the frequency band. The condition of validity of this assumption is one of the more restrictive in SEA theory. Being not the subject of this paper, the reader will be able to find information in reference [19] for example.

The power flow exchanged by the two subsystems, $\Pi_{1-2}$, is the sum of all the individual modeto-mode power flows. Then, one may write:

$$
\Pi_{1-2}=\sum_{p=1}^{N_{1}} \sum_{q=1}^{N_{2}} \Pi_{p q}^{12}=\sum_{p=1}^{N_{1}} \sum_{q=1}^{N_{2}} \beta_{p q}^{12}\left(E_{p}^{1}-E_{q}^{2}\right)
$$

Modal energy equipartition assumption is made:

$$
E_{p}^{1}=e^{1}, \quad \forall p \in\left[1, \ldots, \mathrm{N}_{1}\right] ; \quad \mathrm{E}_{q}^{2}=e^{2}, \quad \forall q \in\left[1, \ldots, \mathrm{N}_{2}\right],
$$

where $e^{1}$ and $e^{2}$ are two constants.

With the orthogonality property of modes, one can write:

$$
\xi_{1}=\sum_{p=1}^{N_{1}} E_{p}^{1}, \quad \xi_{2}=\sum_{q=1}^{N_{2}} E_{q}^{2}
$$

where $\xi_{1}$ and $\xi_{2}$ represent the total energies of subsystem 1 and 2 . 
Therefore, with (10) and (11), one may write:

$$
E_{p}^{1}=\frac{\xi_{1}}{N_{1}}, \quad \mathrm{E}_{q}^{2}=\frac{\xi_{2}}{N_{2}}
$$

that is injected in (9). Finally,

$$
\Pi_{1-2}=\omega_{c} \cdot \eta_{12} \cdot\left(\xi_{1}-\frac{N_{1}}{N_{2}} \cdot \xi_{2}\right),
$$

where the Coupling Loss Factor, $\eta_{12}$, is expressed by

$$
\eta_{12}=\frac{\sum_{p=1}^{N_{1}} \sum_{q=1}^{N_{2}} \beta_{p q}^{12}}{N_{1} \cdot \omega_{c}},
$$

with $\omega_{c}$, central angular frequency of the frequency band of interest.

This final expression permits to calculate the Coupling Loss Factor from the Intermodal Coupling Factors which depend on subsystems modes and on modal coupling coefficients, $\gamma_{p q}^{12}$.

In some cases, one can be interested in developing averaged CLF for an ensemble of substructures. Then, one would have to average the Intermodal Coupling Factors over a population of structures. In reference [1], Lyon gives the mean values of the ICF for a population of structures such that the natural frequencies of each subsystem have their values uniformly probable over the frequency interval of interest, $\Delta \omega$ :

$$
<\beta_{p q}^{12}>_{\omega_{p}^{1} \omega_{q}^{2}}=\frac{\pi}{2 . \Delta \omega}\left(\gamma_{p q}^{12}\right)^{2}
$$

This formula could replace the deterministic ICF in equation (13) in order to obtain the ensemble average Coupling Loss Factor:

$$
<\eta_{12}>_{e}=\frac{\sum_{p=1}^{N_{1}} \sum_{q=1}^{N_{2}}<\beta_{p q}^{12}>_{\omega_{p}^{1} \omega_{q}^{2}}}{N_{1} \cdot \omega_{c}},
$$


One has not yet defined which boundary conditions are necessary to establish equations (5) and how to evaluate the modal coupling coefficients. It is the aim of the next section.

About the equipartition assumption, one can notice that with the approach presented in reference [20] and with the calculation of the Intermodal Coupling Factors by the present approach, it is possible to extend SEA to the case of non modal energy equipartition.

\section{DEFINITION OF SUBSYSTEM MODES AND USE OF THE DUAL MODAL FORMULATION TO OBTAIN C.L.F.}

The approach presented here is not classical for mechanical coupled systems. However, the dual formulation is the standard approach used to study the coupling cavity-structure (see [15], [16], [17]). Therefore, each time that it will be possible, one will make reference to this case to facilitate comprehension.

The present formulation is equally based on the work of Karnopp. Indeed, in reference $[18]^{1}$, he initiated the use of dual formulation (enunciated in [21]) to the coupling of mechanical subsystems and applies the method for the coupling of two rods of identical section. One presents here a generalisation of this approach for the coupling of two continuous mechanical systems.

\subsection{STRUCTURE DESCRIPTION}

One considers two elastic continuous mechanic systems which are rigidly coupled on $S_{\text {Coupling }}$ as shown in figure $3 . V^{l}$ (resp. $V^{2}$ ) represents the volume occupied by the subsystem 1 (resp. subsystem 2). $S_{\text {Clamped }}^{1}$ and $S_{\text {Free }}^{1}$ (resp. $S_{\text {Clamped }}^{2}$ and $S_{\text {Free }}^{2}$ ) are the boundary surfaces with blocked displacements and with free displacements for subsystem 1 (resp. subsystem 2). In a

\footnotetext{
${ }^{1}$ It may be noted that several typing errors and one error of sign are present in the equations of this paper.
} 
first step, free vibration of elastic conservative systems is considered to find modal equations of motion of the coupled subsystems.

In Lagrangian coordinate $x_{i}$, $(\mathrm{i}=1,2,3)$, one defines on the surface of the volume $\mathrm{V}^{1}$ (resp. $\left.\mathrm{V}^{2}\right)$ the unit vector $n_{j}^{1}\left(\operatorname{resp} . n_{j}^{2}\right)$ along the outer normal of the volume. The variables $W_{i}^{1}$ and $W_{i}^{2}$ are supposed to represent displacements in $V^{1}$ and $V^{2}$, respectively. $\sigma_{i j}^{1}$ and $\sigma_{i j}^{2}$ are the stress tensors, $\varepsilon_{i j}^{1}$ and $\varepsilon_{i j}^{2}$ are the strain tensors. And $S_{i j k l}^{1}$ and $S_{i j k l}^{2}$ are the compliance tensors associated to materials of $V^{1}$ and $V^{2}$, respectively. $\rho^{1}$ and $\rho^{2}$ are the mass densities and are supposed independant of time. One studies the dynamical behaviour of the structure between time $t_{0}$ and $t_{1}$.

\section{FIGURE 3}

The continuity conditions on $S_{\text {Coupling }}$ can be expressed by:

$$
\begin{gathered}
\left.W_{i}^{1}=W_{i}^{2} \quad \text { on } S_{\text {Coupling }} \times\right] t_{0}, t_{1}[, \\
\left.\sigma_{i j}^{1} \cdot n_{j}^{1}+\sigma_{i j}^{2} \cdot n_{j}^{2}=0 \quad \text { on } S_{\text {Coupling }} \times\right] t_{0}, t_{1}[.
\end{gathered}
$$

\subsection{DEFINITION OF SUBSTRUCTURES}

For the sub-structuration of the problem, it is now necessary to imagine that one separates these two subsystems and that one prescribes displacements or forces on $S_{\text {Coupling }}$ for each subsystem. One defines $S_{\text {Coupling }}^{1}$ (resp. $S_{\text {Coupling }}^{2}$ ) as the coupling surface $S_{\text {Coupling }}$ in subsystem 1 (resp. subsystem 2).

FIGURE 4 
One chooses to prescribe displacements on $S_{\text {Coupling }}^{1}$ for the subsystem 1 and forces on $S_{\text {Coupling }}^{2}$ for the subsystem 2 (as shown figure 4). Then, for subsystem 1 , one assumes the boundary condition on $S_{\text {Coupling }}^{1}$ to be:

$$
\left.W_{i}^{1}=\bar{W}_{i}^{c} \quad \text { on } S_{\text {Coupling }}^{1} \times\right] t_{0}, t_{1}[\text {. }
$$

And, for subsystem 2 , the boundary condition on $S_{\text {Coupling }}^{2}$ to be:

$$
\left.\sigma_{i j}^{2} \cdot n_{j}^{2}=\vec{F}_{i}^{c} \quad \text { on } S_{\text {Coupling }}^{2} \mathrm{x}\right] t_{0}, t_{1}[
$$

Note: For the cavity - structure coupling, the boundary conditions for the cavity coupled with the structure are the displacements imposed by the structure (like subsystem 1) and the boundary conditions for the structure coupled with the cavity are the pressures imposed by the cavity (like subsystem 2).

Then, from the fundamental equations and principles of continuum mechanics, the linear field equations describing the dynamics of both subsystems can be expressed.

For subsystem 1, the following equations constitute one first problem called problem 1:

- Equations of motion:

$$
\left.\rho^{1} \cdot \frac{\partial^{2} W_{i}^{1}}{\partial t^{2}}=\sigma_{i j, j}^{1} \quad \text { in } V^{1} \times\right] t_{0}, t_{1}[
$$

- Constitutive equations for Hookean linear elastic solid:

$$
\left.\varepsilon_{i j}^{1}=\frac{1}{2} \cdot\left(W_{i, j}^{1}+W_{j, i}^{1}\right)=S_{i j k l}^{1} \sigma_{k l}^{1} \quad \text { in } V^{1} \mathrm{x}\right] t_{0}, t_{1}[.
$$

- Boundary conditions:

$$
\begin{array}{r}
\left.\sigma_{i j}^{1} \cdot n_{j}^{1}=0 \quad \text { on } S_{\text {Free }}^{1} \times\right] t_{0}, t_{1}[, \\
\left.W_{i}^{1}=0 \quad \text { on } S_{\text {Clamped }}^{1} \times\right] t_{0}, t_{1}[, \\
\left.W_{i}^{1}=\bar{W}_{i}^{c} \quad \text { on } S_{\text {Coupling }}^{1} \times\right] t_{0}, t_{1}[.
\end{array}
$$


And for the subsystem 2, one has problem 2:

- Equations of motion:

$$
\left.\rho^{2} \cdot \frac{\partial^{2} W_{i}^{2}}{\partial t^{2}}=\sigma_{i j, j}^{2} \quad \text { in } V^{2} \times\right] t_{0}, t_{1}[
$$

- Constitutive equations for Hookean linear elastic solid:

$$
\left.\varepsilon_{i j}^{2}=\frac{1}{2} \cdot\left(W_{i, j}^{2}+W_{j, i}^{2}\right)=S_{i j k l}^{2} \sigma_{k l}^{2} \quad \text { in } V^{2} \mathrm{x}\right] t_{0}, t_{1}[.
$$

- Boundary conditions:

$$
\begin{gathered}
\left.\sigma_{i j}^{2} \cdot n_{j}^{2}=0 \quad \text { on } S_{\text {Free }}^{2} \times\right] t_{0}, t_{1}[, \\
\left.W_{i}^{2}=0 \quad \text { on } S_{\text {Clamped }}^{2} \times\right] t_{0}, t_{1}[, \\
\left.\sigma_{i j}^{2} \cdot n_{j}^{2}=\vec{F}_{i}^{c} \quad \text { on } S_{\text {Coupling }}^{2} \times\right] t_{0}, t_{1}[.
\end{gathered}
$$

\subsection{VARIATIONAL FORMULATION OF THE PROBLEMS USING REISSNER PRINCIPLE}

For each subsystem, it is possible to express the dynamic problem by using Reissner principle. This formulation will allow us to facilitate the use of the modal expansions.

For problem 1 , the associate Reissner functional $\Psi_{R}^{1}\left(W_{i}^{1}, \sigma_{i j}^{1}\right)$ can be expressed by (see in reference [22] the second form of the Reissner functional):

$$
\begin{gathered}
\Psi_{R}^{1}\left(W_{i}^{1}, \sigma_{i j}^{1}\right): \Omega_{\mathrm{R}}^{1} \mathrm{x} \Sigma_{R}^{1} \rightarrow \mathrm{IR} \\
\left(W_{i}^{1}, \sigma_{i j}^{1}\right) \rightarrow \Psi_{\mathrm{R}}^{1}\left(W_{i}^{1}, \sigma_{i j}^{1}\right) \\
\Psi_{R}^{1}\left(W_{i}^{1}, \sigma_{i j}^{1}\right)=\int_{t_{0}}^{t_{1}}\left\{\int_{V^{1}}\left[\frac{1}{2} \cdot \rho \cdot\left(\frac{\partial^{2} W_{i}^{1}}{\partial t^{2}}\right)+\sigma_{i j, j}^{1} \cdot W_{i}^{1}+\frac{1}{2} \cdot \sigma_{i j}^{1} \cdot S_{i j k l}^{1} \cdot \sigma_{k l}^{1}\right] d V-\int_{S_{\text {Coupling }}^{1}} \bar{W}_{i}^{c} \cdot \sigma_{i j}^{1} \cdot n_{j}^{1} \cdot d S\right\} d t .
\end{gathered}
$$

The associate admissible spaces are:

$$
\begin{gathered}
\Omega_{R}^{1}\left(V^{1} x\right] t_{0}, t_{1}[)=\left\{\mathrm{W}_{i}^{1} / \mathrm{W}_{i}^{1} \in \mathrm{H}^{1}\left(V^{1}\right) ; \mathrm{W}_{\mathrm{i}}^{1}=0 / \mathrm{S}_{\text {Clamped }}^{1}\right\}, \\
\Sigma_{R}^{1}\left(V^{1} x\right] t_{0}, t_{1}[)=\left\{\sigma_{i j}^{1} / \sigma_{i j}^{1}=\sigma_{j i}^{1} ; \quad \sigma_{i j}^{1} \in L^{2}\left(V^{1}\right) ; \quad \sigma_{i j, j}^{1} \in L^{2}\left(V^{1}\right) ; \sigma_{i j}^{1} \cdot n_{j}=0 / S_{\text {Free }}^{1}\right\} .
\end{gathered}
$$


The problem constituted by equations (21-25) can be replaced by searching $W_{i}^{1}$ and $\sigma_{i j}^{1}$ in their respective admissible spaces $\Omega_{R}^{1}$ and $\Sigma_{\mathrm{R}}^{1}$ such that they render the functional $\Psi_{R}^{1}\left(W_{i}^{1}, \sigma_{i j}^{1}\right)$ stationary.

In the same way, for problem 2 , the Reissner functional $\Psi_{R}^{2}\left(W_{i}^{2}, \sigma_{i j}^{2}\right)$ can be expressed by (see in reference [22] the first form of the Reissner functional):

$$
\begin{gathered}
\Psi_{R}^{2}\left(W_{i}^{2}, \sigma_{i j}^{2}\right): \Omega_{\mathrm{R}}^{2} \mathrm{x} \Sigma_{R}^{2} \rightarrow \mathrm{IR} \\
\left(W_{i}^{2}, \sigma_{i j}^{2}\right) \rightarrow \Psi_{\mathrm{R}}^{2}\left(W_{i}^{2}, \sigma_{i j}^{2}\right) \\
\Psi_{R}^{2}\left(W_{i}^{2}, \sigma_{i j}^{2}\right)=\int_{t_{0}}^{t_{1}}\left\{\int_{V^{2}}\left[\frac{1}{2} \cdot \rho \cdot\left(\frac{\partial^{2} W_{i}^{2}}{\partial t^{2}}\right)-\sigma_{i j}^{2} \cdot \frac{1}{2} \cdot\left(W_{i, j}^{2}+W_{j, i}^{2}\right)+\frac{1}{2} \cdot \sigma_{i j}^{2} \cdot S_{i j k l}^{2} \cdot \sigma_{k l}^{2}\right] d V-\int_{S_{\text {Coupling }}^{2}} \bar{F}_{i}^{c} \cdot W_{i}^{2} d S\right\} d t .
\end{gathered}
$$

And the associate admissible spaces:

$$
\begin{gathered}
\Omega_{R}^{2}\left(V^{2} x\right] t_{0}, t_{1}[)=\left\{\mathrm{W}_{i}^{2} / \mathrm{W}_{i}^{2} \in \mathrm{H}^{1}\left(V^{2}\right) ; \mathrm{W}_{\mathrm{i}}^{2}=0 / \mathrm{S}_{\text {Clamped }}^{1}\right\}, \\
\sum_{R}^{2}\left(V^{2} x\right] t_{0}, t_{1}[)=\left\{\sigma_{i j}^{2} / \sigma_{i j}^{2}=\sigma_{j i}^{2} ; \quad \sigma_{i j}^{2} \in L^{2}\left(V^{2}\right) ; \quad \sigma_{i j}^{2} \cdot n_{j}=0 / S_{\text {Free }}^{1}\right\} .
\end{gathered}
$$

In this case, the problem constituted by equations (26-30) can be replaced by searching $W_{i}^{2}$ and $\sigma_{i j}^{2}$ in their respective admissible spaces $\Omega_{R}^{2}$ and $S_{R}^{2}$ such that they render the functional $\Psi_{R}^{2}\left(W_{i}^{2}, \sigma_{i j}^{2}\right)$ stationary.

\subsection{MODES OF UNCOUPLED SUBSYSTEMS}

\subsubsection{Definition of subsystem modes}

In a first step, modal expansion will be used to solve separately the two previous variational problems. The sets of mode shapes which will be considered, generate subspaces of admissible 
spaces associated to each variational problem. Then, the approximate solutions of these problems will be found considering these subspaces. The definition of the modes of each subsystem must be judicious in order to permit to link the two problems in a second step. Indeed, the coupling of the problems will be possible if modal expansion of the stress field of subsystem 1 allows one to determinate the force excitation of subsystem 2, and 'inversely', if modal expansion of the displacement field of subsystem 2 allows one to calculate the displacement excitation of subsystem 1. The subspaces of admissible spaces, and by consequence, the mode' definitions must be chosen adequately .

Then, modes of subsystem 1 are defined with blocked displacements on $S_{\text {Coupling }}^{1}$ (as shown figure 5 (a)). One calls them, the modes of the uncoupled - blocked subsystem or shortly the blocked modes. These modes respect the admissibility conditions on stresses for the problem 1. (In the case of cavity - structure coupling, the modes of the cavity are the blocked modes because they are determined with rigid walls.)

On the other hand, modes of subsystem 2 are calculated with null stresses on $S_{\text {Coupling }}^{2}$ (as shown figure 5 (b)). One calls them, the modes of the uncoupled - free subsystem or free modes. These modes respect the kinematic admissible conditions for the problem 2. (They correspond to the modes of the in-vacuo structure in the case of cavity - structure coupling.)

\section{FIGURE 5}

In SEA point of view, it is well known that the coupling between subsystems must be weak, that is to say those input mobilities on the coupling surface of the two subsystems must be 'very' different. Then, the choice of 'which subsystems with which boundary conditions?' find its answer in weak coupling's definition in reference [23]: "Under conditions of weak coupling, the system modes are 'localised' in the sense that they closely resemble in natural frequency and shape the 
modes of the uncoupled subsystems (given the appropriate boundary conditions), ...". Thus, the modes of the uncoupled subsystems must be chosen such they resemble the physical modes of the global structures. In the case of weak coupling, there is an impedance rupture between the two subsystems that leads one subsystem to vibrate rather freely and the other one to vibrate as it is clamped. Free modes are, then, the physical modes for the subsystem which vibrates freely, and the blocked modes are the physical modes for the other subsystem.

\subsubsection{Calculations and properties of modes}

The eigenvalue problems respected independently by each field can be obtained from equations of motion, constitutive law and boundary conditions expressed in mixed variables:

- For the displacement field of subsystem $\alpha(\alpha=1,2)$, the eigenvalue problem called primal problem can be written after a separation of time and space, $W_{i}^{\alpha}(M, t)=\tilde{W}_{i}^{\alpha}(M) \cdot e^{j \omega^{\alpha} t}$ :

$$
\left\{\begin{array}{l}
-\rho^{\alpha} \cdot\left(\omega^{\alpha}\right)^{2} \tilde{W}_{i}^{\alpha}=\frac{1}{2} \cdot S_{i j k l}^{\alpha} \cdot\left(\tilde{W}_{k, l}^{\alpha}+\tilde{W}_{l, k}^{\alpha}\right)_{, j} \text { in } V^{\alpha}, \\
+ \text { Boundary conditions. }
\end{array}\right.
$$

- And for the stress field of subsystem $\alpha$, the eigenvalue problem called dual problem can be written with $\sigma_{i j}^{\alpha}(M, t)=\tilde{\sigma}_{i j}^{\alpha}(M) \cdot e^{j \omega^{\alpha} t}$ :

$$
\left\{\begin{array}{l}
-\rho^{\alpha} \cdot\left(\omega^{\alpha}\right)^{2} \cdot \tilde{\sigma}_{i j}^{\alpha}=S_{i j k l}^{\alpha-1}\left(\tilde{\sigma}_{k m, m, l}^{\alpha}+\tilde{\sigma}_{l m, m, k}^{\alpha}\right) \text { in } V^{\alpha}, \\
+ \text { Boundary conditions. }
\end{array}\right.
$$

These two problems (primal and dual) are extracted from the same problem expressed in mixed variables. In general, the problem in mixed variables is respected by the couples of solutions $\left(\omega^{\alpha}, \tilde{W}_{i}^{\alpha}, \tilde{\sigma}_{i j}^{\alpha}\right)$. However, one can notice that one solution of the dual problem having a null eigenvalue can exist and do not have equivalence in the primal problem. Then, this solution does not appear in mixed variables. For a cavity, it corresponds to the Helmholtz mode that is found in 
term of pressure and not in term of displacement. This solution having a null eigenfrequency will have a negligible contribution in the modal expansion (because non-resonant) as soon as others resonant modes participate to the response. The CLF calculation taking only into account the resonant modes in a frequency band, the Helmoltz mode contribution can be neglected. Thus, one will use the problem in mixed variables to define the stress and displacement shapes of each mode.

Let us defined:

- $\quad$ for the uncoupled - blocked subsystem 1:

$$
\begin{gathered}
\left\{\begin{array}{l}
\omega_{p}^{1}: \text { the natural angular frequency of mode } \mathrm{p}, \\
\tilde{W}_{i}^{1 p}(M): \text { the mode } \mathrm{p} \text { displacements, and } \\
\tilde{\sigma}_{i j}^{1 p}(M): \text { the mode p stresses, }
\end{array}\right. \\
\text { with the following equations }\left(\text { for } \omega_{p}^{1} \neq 0\right): \\
-\rho^{1} \cdot\left(\omega_{p}^{1}\right)^{2} \cdot \tilde{W}_{i}^{1 p}=\tilde{\sigma}_{i j, j}^{1 p} \quad \text { in } V^{1}, \\
\frac{1}{2} \cdot\left(\tilde{W}_{i, j}^{1 p}+\tilde{W}_{j, i}^{1 p}\right)=S_{i j k l}^{1} \tilde{\sigma}_{k l}^{1 p} \quad \text { in } V^{1}, \\
\tilde{\sigma}_{i j}^{1 p} . n_{j}^{1}=0 \text { on } S_{\text {Free }}^{1}, \quad \tilde{W}_{i}^{1 p}=0 \text { on } S_{\text {Clamped }}^{1}, \quad \tilde{W}_{i}^{1 p}=0 \text { on } S_{\text {Coupling }}^{1},
\end{gathered}
$$

- and for the uncoupled - free subsystem 2:

$$
\begin{aligned}
& \qquad \begin{array}{l}
\omega_{q}^{2}: \text { the natural angular frequency of mode q, } \\
\tilde{W}_{i}^{2 q}(M): \text { the mode q displacements, and } \\
\tilde{\sigma}_{i j}^{2 q}(M): \text { the mode q stresses, }
\end{array} \\
& \text { with the following equations }\left(\text { for } \omega_{q}^{2} \neq 0\right): \\
& -\rho^{2} \cdot\left(\omega_{q}^{2}\right)^{2} \cdot \tilde{W}_{i}^{2 q} \cdot=\tilde{\sigma}_{i j, j}^{2 q} \quad \text { in } V^{2}, \\
& \frac{1}{2} \cdot\left(\tilde{W}_{i, j}^{2 q}+\tilde{W}_{j, i}^{2 q}\right)=S_{i j k l}^{2} \tilde{\sigma}_{k l}^{2 q} \quad \text { in } V^{2}, \\
& \tilde{\sigma}_{i j}^{2 q} \cdot n_{j}^{2}=0 \quad \text { on } S_{\text {Free }}^{2}, \quad \tilde{W}_{i}^{2 q}=0 \quad \text { on } S_{\text {Clamped }}^{2}, \quad \tilde{\sigma}_{i j}^{2 q} \cdot n_{j}^{2}=0 \quad \text { on } S_{\text {Coupling }}^{2} .
\end{aligned}
$$


These modes have the following orthogonality properties (see [24]) for subsystem $\alpha(\alpha=1,2)$ :

$$
\begin{gathered}
\int_{V^{\alpha}} \omega_{p}^{\alpha} \cdot \omega_{q}^{\alpha} \cdot \rho \cdot \tilde{W}_{i}^{\alpha p} \cdot \tilde{W}_{i}^{\alpha q} \cdot d V=K_{p}^{\alpha} \cdot \delta_{p q}, \\
\int_{V^{\alpha}} \tilde{\sigma}_{i j}^{\alpha p} \cdot S_{i j k l}^{\alpha} \cdot \tilde{\sigma}_{k l}^{\alpha q} \cdot d V=K_{p}^{\alpha} \cdot \delta_{p q}, \\
\int_{V^{\alpha}} \tilde{\sigma}_{i j, j}^{\alpha p} \cdot \tilde{W}_{i}^{\alpha q} \cdot d V=K_{p}^{\alpha} \cdot \delta_{p q}, \\
\int_{V^{\alpha}} \tilde{\sigma}_{i j}^{\alpha p} \cdot \frac{1}{2} \cdot\left(\tilde{W}_{i, j}^{\alpha q}+\tilde{W}_{j, i}^{\alpha q}\right) \cdot d V=K_{p}^{\alpha} \cdot \delta_{p q},
\end{gathered}
$$

where $K_{p}^{\alpha}$ is the modal stiffness of mode p of subsystem $\alpha$, and $\delta_{p q}$ is the Kronecker symbol $\left(\delta_{p q}=0\right.$ if $\mathrm{p} \neq \mathrm{q} ; \delta_{p q}=1$ if $\left.\mathrm{p}=\mathrm{q}\right)$.

The natural angular frequencies can be expressed by:

$$
\omega_{p}^{\alpha}=\sqrt{\frac{K_{p}^{\alpha}}{M_{p}^{\alpha}}}, \quad \alpha=1,2
$$

where $M_{p}^{\alpha}$ is the modal mass of mode p of subsystem $\alpha$.

Note: The blocked modes of subsystem 1 respect the admissibility conditions on stresses of the variational problem 1 . Thus, the set of the stress mode shapes $\left\{\tilde{\sigma}_{i j}^{1 p}(M), \mathrm{p}=1.2 \ldots,+\infty\right\}$ (taking into account the stress mode of null frequency if it exists) constitutes a base of the admissible space $\Sigma_{R}^{1}$. In a same way, the uncoupled - free modes of subsystem 2 respect the kinematic admissible conditions of the variational problem 2. The set of the displacement mode shapes $\left\{\tilde{W}_{i}^{2 q}(M), \mathrm{q}=1.2 \ldots,+\infty\right\}$ constitutes a base of $\Omega_{R}^{2}$ (the displacement kinematically admissible space). This will allow one to calculate the stress field for subsystem 1 and the displacement field for subsystem 2 on the coupling boundary from the modal expansion. 


\subsection{MODAL EXPANSION OF THE SOLUTION}

Expanding displacements and stresses of each subsystem in the modal bases, one can write:

$$
\begin{aligned}
& W_{i}^{1}(M, t)=\sum_{n=1}^{\infty} a_{n}^{1}(t) \cdot \tilde{W}_{i}^{1 n}(M), \sigma_{i j}^{1}(M, t)=\sum_{m=1}^{\infty} b_{m}^{1}(t) \cdot \tilde{\sigma}_{i j}^{1 m}(M), \\
& W_{i}^{2}(M, t)=\sum_{r=1}^{\infty} a_{r}^{2}(t) \cdot \tilde{W}_{i}^{2 r}(M), \sigma_{i j}^{2}(M, t)=\sum_{s=1}^{\infty} b_{s}^{2}(t) \cdot \tilde{\sigma}_{i j}^{2 s}(M) .
\end{aligned}
$$

The expansion allows one to find the weak solutions of the two problems (21-25), (26-30) by using Reissner Principle and subspaces of admissible spaces. These solutions are weak solutions because the subspace generated by mode shapes will not permit strictly verification of equation (25) for problem 1 and equation (30) for problem 2.

Introducing expansions $(50,51)$ into the variational principles $(31,34)$, and using the modes' orthogonality properties (45-48), the solution of each problem is then obtained by finding modal amplitudes that render stationary the Reissner's Functional. That is to say modal amplitudes satisfying Euler equation (52) associated to $a_{n}^{1}, b_{m}^{1}$, (resp. $\left.a_{p}^{2}, b_{q}^{2}\right)$ for subsystem 1 (resp. subsystem 2):

$$
/ q_{i}: \frac{\partial}{\partial q_{i}}\left(F\left(q_{i}(t), \dot{q}_{i}(t)\right)\right)-\frac{d}{d t}\left(\frac{\partial}{\partial \dot{q}_{i}}\left(F\left(q_{i}(t), \dot{q}_{i}(t)\right)\right)\right)=0,
$$

where $F\left(q_{i}(t), \dot{q}_{i}(t)\right)$ is the considered functional which depends on $q_{i}(t), \dot{q}_{i}(t)$.

One obtains finally, for subsystem 1 :

$$
\begin{gathered}
/ a_{p}^{1}:-\frac{K_{p}^{1}}{\left(\omega_{p}^{1}\right)^{2}} \cdot \ddot{a}_{p}^{1}(t)-K_{p}^{1} \cdot b_{p}^{1}(t)=0, \\
/ b_{p}^{1}(t):-K_{p}^{1} \cdot a_{p}^{1}(t)+K_{p}^{1} \cdot b_{p}^{1}(t)-\int_{S_{\text {Coupling }}^{1}} \bar{W}_{i}^{c} \cdot \tilde{\sigma}_{i j}^{1 p} \cdot n_{j}^{1} d S=0,
\end{gathered}
$$


and, for subsystem 2:

$$
\begin{gathered}
/ a_{q}^{2}:-\frac{K_{q}^{2}}{\left(\omega_{q}^{2}\right)^{2}} \cdot \ddot{a}_{q}^{2}(t)-K_{q}^{2} \cdot b_{q}^{2}(t)+\int_{S_{\text {Coupling }}^{2}} \bar{F}_{i}^{c} \cdot \tilde{W}_{i}^{2 q} d S=0, \\
/ b_{q}^{2}(t): \quad-K_{q}^{2} \cdot a_{q}^{2}(t)+K_{q}^{2} \cdot b_{q}^{2}(t)=0
\end{gathered}
$$

According to dual formulation used by Karnopp [18], one must describe the behaviour of subsystem 1 with stress modal amplitudes, $b_{p}^{1}(t)$, and subsystem 2 with displacement modal amplitudes, $a_{q}^{2}(t)$. It is analogous to the coupling cavity - structure where the pressure is the descriptive variable for the subsystem with blocked modes (cavity), and the displacements are the descriptive variables for the subsystem with free modes (structure). Therefore, combining time second derivative of equation (54) with (53), and (55) with (56), give:

$$
\begin{gathered}
\ddot{b}_{p}^{1}(t)+\left(\omega_{p}^{1}\right)^{2} \cdot b_{p}^{1}(t)=\frac{1}{\left(\omega_{p}^{1}\right)^{2} \cdot M_{p}^{1}} \cdot \int_{S_{\text {Coupling }}^{1}} \ddot{\bar{W}}_{i}^{c} \cdot \tilde{\sigma}_{i j}^{1 p} \cdot n_{j}^{1} d S, \\
\ddot{a}_{q}^{2}(t)+\left(\omega_{q}^{2}\right)^{2} \cdot a_{q}^{2}(t)=\frac{1}{M_{q}^{2}} \cdot \int_{S_{\text {Coupling }}^{2}} \bar{F}_{i}^{c} \cdot \tilde{W}_{i}^{2 q} d S .
\end{gathered}
$$

\subsection{FREE VIBRATIONS OF COUPLED SUBSYSTEMS}

Equations (57) and (58) describe the coupling through specified displacements on subsystem 1 and forces on subsystem 2. To express coupling conditions, one has to say that the specified force acting on subsystem 2 is the opposite of stress boundary vector of subsystem 1 (see equation (18)), and, the prescribed displacements on subsystem 1 is the displacements of subsystem 2 on the coupling surface (see equation (17)). It is now easy to express the accelerations and the forces on the coupling surface by using modal expansions:

$$
\begin{gathered}
\bar{F}_{i}^{c}=-\sum_{m=1}^{\infty} b_{m}^{1}(t) \cdot \tilde{\sigma}_{i j}^{1 m} \cdot n_{j}^{1} \text { on } S_{\text {Coupling }}, \\
\ddot{\bar{W}}_{i}^{c}=\sum_{r=1}^{\infty} \ddot{a}_{r}^{2}(t) \cdot \tilde{W}_{i}^{2 r} \text { on } S_{\text {Coupling }} .
\end{gathered}
$$


Injecting (60) in (57), and (59) in (58), one obtains the system of equations,

$$
\begin{cases}\ddot{b}_{p}^{1}(t)+\left(\omega_{p}^{1}\right)^{2} \cdot b_{p}^{1}(t)=\frac{1}{\left(\omega_{p}^{1}\right)^{2} \cdot M_{p}^{1}} \cdot \sum_{r=1}^{\infty} \ddot{a}_{r}^{2}(t) \cdot \int_{S_{\text {Coupling }}} \cdot \tilde{W}_{i}^{2 r} \cdot \tilde{\sigma}_{i j}^{1 p} \cdot n_{j}^{1} d S, & \forall p \in[1, \ldots, \infty[, \\ \ddot{a}_{q}^{2}(t)+\left(\omega_{q}^{2}\right)^{2} \cdot a_{q}^{2}(t)=-\frac{1}{M_{q}^{2}} \quad \sum_{m=1}^{\infty} b_{m}^{1}(t) \cdot \int_{S_{\text {Coupling }}} \cdot \tilde{W}_{i}^{2 q} \cdot \tilde{\sigma}_{i j}^{1 m} \cdot n_{j}^{1} d S, & \forall \mathrm{q} \in[1, \ldots, \infty[.\end{cases}
$$

To have equations analogous to equations (5), it is necessary to carry out the change of variable:

$$
b_{p}^{1}(t)=\dot{c}_{p}^{1}(t)
$$

For the cavity - structure problem, the pressure is replaced by the acoustic - fluid velocity potential, which, with the Euler law in fluid medium, represents a change of variable similar to (62).

Finally, one obtains the equations governing free vibrations of coupled substuctures:

$$
\begin{cases}\ddot{c}_{p}^{1}(t)+\left(\omega_{p}^{1}\right)^{2} \cdot c_{p}^{1}(t)=\frac{1}{\left(\omega_{p}^{1}\right)^{2} \cdot M_{p}^{1}} \cdot \sum_{r=1}^{\infty} \dot{a}_{r}^{2}(t) \cdot \int_{S_{\text {Coupling }}} \cdot \tilde{W}_{i}^{2 r} \cdot \tilde{\sigma}_{i j}^{1 p} \cdot n_{j}^{1} d S, & \forall p \in[1, \ldots, \infty[, \\ \ddot{a}_{q}^{2}(t)+\left(\omega_{q}^{2}\right)^{2} \cdot a_{q}^{2}(t)=-\frac{1}{M_{q}^{2}} \quad \sum_{m=1}^{\infty} \dot{c}_{m}^{1}(t) \cdot \int_{S_{\text {Coupling }}} \cdot \tilde{W}_{i}^{2 q} \cdot \tilde{\sigma}_{i j}^{1 m} \cdot n_{j}^{1} d S, & \forall \mathrm{q} \in[1, \ldots, \infty[.\end{cases}
$$

One notices, and it is an advantage of the dual formulation, that compared to displacement formulation there are not 'direct couplings' between modes of the same subsystem (see [25]). Indeed, from a formal point of view, mode 'p' of subsystem 1 is coupled with the modes of subsystem 2 but it is not directly coupled with the others modes of subsystem 1 . In the same way, mode 'q' of subsystem 2 is coupled with the modes of subsystem 1 but it is not coupled with the other modes of subsystem 2.

\subsection{FORCED VIBRATION OF COUPLED SUBSYSTEMS}

By introducing excitation and damping in equations (63), one get the system of equations describing the forced response of the coupled subsystems from the modal amplitudes of the modes of uncoupled subsystems: 


$$
\left\{\begin{array}{c}
\ddot{c}_{p}^{1}(t)+\Delta_{p}^{1} \cdot \dot{c}_{p}^{1}(t)+\left(\omega_{p}^{1}\right)^{2} \cdot c_{p}^{1}(t)-\frac{1}{\left(\omega_{p}^{1}\right)^{2} \cdot M_{p}^{1}} \cdot \sum_{r=1}^{\infty} \dot{a}_{r}^{2}(t) \cdot \int_{S_{\text {Coupling }}} \cdot \tilde{W}_{i}^{2 r} \cdot \tilde{\sigma}_{i j}^{1 p} \cdot n_{j}^{1} d S=\frac{F_{p}^{1}}{\left(\omega_{p}^{1}\right)^{2} \cdot M_{p}^{1}}, \\
\forall p \in[1, \ldots, \infty[, \\
\ddot{a}_{q}^{2}(t)+\Delta_{q}^{2} \cdot \dot{a}_{q}^{2}(t)+\left(\omega_{q}^{2}\right)^{2} \cdot a_{q}^{2}(t)+\frac{1}{M_{q}^{2}} \quad \sum_{m=1}^{\infty} \dot{c}_{m}^{1}(t) \cdot \int_{S_{\text {Coupling }}} \cdot \tilde{W}_{i}^{2 q} \cdot \tilde{\sigma}_{i j}^{1 m} \cdot n_{j}^{1} d S=\frac{F_{q}^{2}}{M_{q}^{2}}, \\
\forall \mathrm{q} \in[1, \ldots, \infty[,
\end{array}\right.
$$

where one has introduced modal viscous damping through the modal damping bandwidths $\Delta_{p}^{1}$ and $\Delta_{q}^{2}$, and external excitations through the generalised terms $F_{p}^{1}$ and $F_{q}^{2}$.

To identify the modal coupling coefficient as defined in the basic model of two coupled oscillators, one considers only the coupling of the $\mathrm{p}^{\text {th }}$ mode of subsystem 1 and the $\mathrm{q}^{\text {th }}$ mode of subsystem 2:

$$
\left\{\begin{array}{r}
\ddot{c}_{p}^{1}(t)+\Delta_{p}^{1} \cdot \dot{c}_{p}^{1}(t)+\left(\omega_{p}^{1}\right)^{2} \cdot c_{p}^{1}(t) \\
-\sqrt{\frac{M_{q}^{2}}{\left(\omega_{p}^{1}\right)^{2} \cdot M_{p}^{1}}} \cdot\left[\frac{1}{\sqrt{\left(\omega_{p}^{1}\right)^{2} \cdot M_{p}^{1} \cdot M_{q}^{2}}} \int_{S_{\text {Coup ping }}} \int_{W_{i}^{2 q}}^{2 q} \cdot \tilde{\sigma}_{i j}^{1 p} \cdot n_{j}^{1} d S\right] \cdot \dot{a}_{q}^{2}(t)=\frac{L_{1 p q}}{\left(\omega_{p}^{1}\right)^{2} \cdot M_{p}^{1}}, \\
\quad \forall(p, q) \in\left(\left[1, \ldots, \infty[)^{2},\right.\right. \\
+\sqrt{\frac{\left(\omega_{p}^{1}\right)^{2} \cdot M_{p}^{1}}{M_{q}^{2}}} \cdot\left[\frac{1}{\sqrt{\left(\omega_{p}^{1}\right)^{2} \cdot M_{p}^{1} \cdot M_{q}^{2}}} \int_{S_{\text {Coupling }}}\left[\tilde{W}_{i}^{2 q} \cdot \tilde{\sigma}_{i j}^{1 p} \cdot n_{j}^{1} d S\right] \dot{c}_{p}^{1}(t)=\frac{L_{2 p q}}{M_{q}^{2}},\right.
\end{array}\right.
$$

where $L_{1 p q}$ and $L_{2 p q}$ contain the generalised 'forces' and the interaction 'forces' with all the others modes.

One deduces directly by comparison with (6) the modal coupling coefficient between the $\mathrm{p}^{\text {th }}$ mode and the $\mathrm{q}^{\text {th }}$ mode:

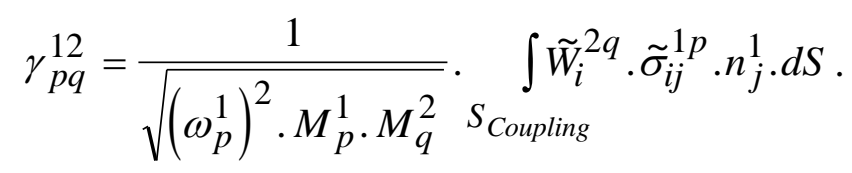


Physically, it is important to notice that the integral represents the interaction modal work exchanged by the $\mathrm{p}^{\text {th }}$ blocked mode of subsystem 1 and the $\mathrm{q}^{\text {th }}$ free mode of subsystem 2 . One notes the interaction modal work by:

$$
\mathbf{W}_{p q}^{12}=\int_{S_{\text {Coupling }}} \tilde{W}_{i}^{2 q} \cdot \tilde{\sigma}_{i j}^{1 p} \cdot n_{j}^{1} \cdot d S .
$$

This is demonstrated in the general case of three dimensional continuum mechanical subsystems, and thus can be accepted as a basic principle that can be applied in simplified models of beams, plates and shells. In these cases, one has to consider the work associated to dual variables introduced in 1D or 2D models (force - displacement, moment - rotation, etc.).

\subsection{CALCULATION OF COUPLING LOSS FACTORS}

Combining equations (8), (14), (66) and (67), one obtains a general expression allowing us to calculate the Coupling Loss Factors from subsystem modal information:

$$
\eta_{12}=\frac{1}{N_{1} \cdot \omega_{c}} \cdot \sum_{p=1}^{N_{1}} \sum_{q=1}^{N_{2}}\left[\frac{\left(\mathbf{W}_{p q}^{12}\right)^{2}}{\left(\omega_{p}^{1}\right)^{2} \cdot M_{p}^{1} \cdot M_{q}^{2}}\left(\frac{\left[\Delta_{p}^{1} \cdot\left(\omega_{q}^{2}\right)^{2}+\Delta_{q}^{2} \cdot\left(\omega_{p}^{1}\right)^{2}\right]}{\left(\left(\omega_{p}^{1}\right)^{2}-\left(\omega_{q}^{2}\right)^{2}\right)^{2}+\left(\Delta_{p}^{1}+\Delta_{q}^{2}\right)\left(\Delta_{p}^{1} \cdot\left(\omega_{q}^{2}\right)^{2}+\Delta_{q}^{2} \cdot\left(\omega_{p}^{1}\right)^{2}\right)}\right)\right] .
$$

\subsection{ENERGY PROPERTIES}

The equation system (64) can be interpreted as the coupling between a set of oscillators associated to subsystem 1 with another set of oscillators associated to subsystem 2. One is interested here in evaluating the energy properties of these associated oscillators. In a first step, one establishes the relations between subsystem energies and modal energies, and in a second step one evaluates the links between the energies associated to amplitudes $a_{q}^{2}(t)$ and $c_{p}^{1}(t)$, and the modal energies.

\subsubsection{Subsystem energy and modal energy}

- The instantaneous kinetic energy of subsystem $\alpha(\alpha=1,2)$ is expressed by :

$$
E_{K}^{\alpha}=\int_{V^{\alpha}} \frac{1}{2} \cdot \rho \cdot\left(\frac{\partial W_{i}^{\alpha}}{\partial t}\right)^{2} d V
$$


Using the displacement modal expansion and taking into account the modal orthogonality property (45), gives

$$
E_{K}^{\alpha}(t)=\frac{1}{2} \sum_{n=1}^{\infty} M_{n}^{\alpha} \cdot\left(\dot{a}_{n}^{\alpha}(t)\right)^{2}
$$

therefore,

$$
E_{K}^{\alpha}(t)=\sum_{n=1}^{\infty} E_{K}^{\alpha n}(t)
$$

where $E_{K}^{\alpha n}(t)=\frac{1}{2} \cdot M_{n}^{\alpha} \cdot\left(\dot{a}_{n}^{\alpha}(t)\right)^{2}$ is the modal kinetic energy of mode $\mathrm{n}$ of subsystem $\alpha$.

- With the same manner, the instantaneous potential energy of subsystem $\alpha$ is expressed by:

$$
E_{P}^{\alpha}(t)=\frac{1}{2} \int_{V^{\alpha}} \sigma_{i j}^{\alpha} \cdot S_{i j k l}^{\alpha} \cdot \sigma_{k l}^{\alpha} d V
$$

Using the stress modal expansion, and taking into account the modal orthogonality property (46), gives finally

$$
E_{P}^{\alpha}(t)=\frac{1}{2} \sum_{n=1}^{\infty} K_{n}^{\alpha} \cdot\left(b_{n}^{\alpha}(t)\right)^{2}
$$

Therefore,

$$
E_{P}^{\alpha}(t)=\sum_{n=1}^{\infty} E_{P}^{\alpha n}(t)
$$

where $E_{P}^{\alpha n}(t)=\frac{1}{2} \cdot K_{n}^{\alpha} .\left(b_{n}^{\alpha}(t)\right)^{2}$ is the modal potential energy of mode $\mathrm{n}$ of subsystem $\alpha$.

In conclusion, the total energy of a subsystem is equal to the sum of the modal total energies of all modes.

\subsubsection{Energy properties associated to amplitudes $a_{q}^{2}(t)$ and $c_{p}^{1}(t)$}

From the form of (64), the modal amplitudes $a_{q}^{2}(t)$ can be associated to the displacement amplitude of an oscillator whose its mass is the generalised mass and its stiffness is the generalised stiffness of mode q. It is well known that the kinetic (resp. potential) energy of the oscillator 
represents the mode q kinetic energy (resp. potential energy, taking into account (56)). Thus the total energy of the oscillator is the modal total energy.

For subsystem 1, the parameters, which must be associated to the oscillator, are not classical (as for subsystem 2). Calculations are then necessary to identify what are represented by kinetic energy and potential energy of the corresponding oscillator:

\section{FIGURE 6}

One notes $M$, the mass, $K$, the stiffness and $y(t)$, the amplitude of the oscillator. By identification from the form of equation (64), one associate the modal parameters of the $\mathrm{p}^{\text {th }}$ blocked mode of subsystem 1 to the oscillator parameters as shown in figure 6: The oscillator mass, $M$, is equal to the modal stiffness, $K_{p}^{1}$; And the oscillator stiffness, $K$, is equal to $\frac{\left(K_{p}^{1}\right)^{2}}{M_{p}^{1}}$.

The kinetic energy of the oscillator at any time can be expressed by:

$$
E_{K}(t)=\frac{1}{2} \cdot M \cdot(\dot{y}(t))^{2}=\frac{1}{2} \cdot K_{p}^{1} \cdot\left(\dot{c}_{p}^{1}(t)\right)^{2}
$$

By introducing relation (62), one obtains:

$$
E_{K}(t)=\frac{1}{2} \cdot K_{p}^{1} \cdot\left(b_{p}^{1}(t)\right)^{2}
$$

Thus, the kinetic energy of the associated oscillator is the potential energy of the $\mathrm{p}^{\text {th }}$ blocked mode.

For the potential energy of the oscillator, one can write:

$$
E_{P}(t)=\frac{1}{2} \cdot K \cdot(y(t))^{2}=\frac{1}{2} \cdot \frac{\left(K_{p}^{1}\right)^{2}}{M_{p}^{1}} \cdot\left(c_{p}^{1}(t)\right)^{2}
$$


Injecting (62) in (53), then calculating the integral over the time (taking into account that one considers stationary motion), one has:

$$
c_{p}^{1}(t)=-\frac{M_{p}^{1}}{K_{p}^{1}} \cdot \dot{a}_{p}^{1}(t)
$$

Injecting in (77), gives:

$$
E_{P}(t)=\frac{1}{2} \cdot M_{p}^{1} \cdot\left(\dot{a}_{p}^{1}(t)\right)^{2}
$$

Thus, the potential energy of the associated oscillator is the kinetic energy of the $\mathrm{p}^{\text {th }}$ blocked mode. It is, however, important to notice that the total energy of the associated oscillator is equal to the total energy of the $\mathrm{p}^{\text {th }}$ blocked modes.

\section{CLF CALCULATION WITH FEM DATA}

\subsection{INTRODUCTION}

The goal of the present approach is to apply SEA to complicated substructures. In that case only a finite element model of each subsystem can be performed. The advantages of the proposed method to calculate CLF in this case are:

- Subsystem boundary conditions are clearly defined (the uncoupled modes are clearly defined).

- Heterogeneous subsystems having three dimensional vibration motions can be treated without any difficulty. This can be quite difficult to do with the classical numerical experiment and inverse SEA technique due to the choice of the position and type of force which should be applied.

- The present technique has a short computing time, because the resolution of the equation of motion is not necessary. The CLF are calculated directly from the coefficients of modal equations. 


\subsection{INTERACTION MODAL WORK FOR DISCRETIZED SYSTEM}

In our approach, CLF are calculated from the interaction modal work of couple of modes. For a FEM discretized system, one can determine the expression of the interaction modal work directly from the nodal variables. For the node i, the displacement variables are the three displacements $\left(u_{k}^{i}, k=1,2,3\right)$ and the three rotations $\left(u_{k}^{i}, k=4,5,6\right)$; And the dual variables are the three forces $\left(f_{k}^{i}, k=1,2,3\right)$, and the three moments $\left(f_{k}^{i}, k=4,5,6\right)$.

One considers a system composed of two vibrating subsystems discretized by Finite Element and coupled together. When they are coupled, the two subsystems have some common nodes called 'coupling node set' on the coupling boundary. As in section 3, one supposes that subsystem 1 is the uncoupled - blocked subsystem and that subsystem 2 is the uncoupled - free subsystem. Then, subsystem 1 must be described by the nodal forces $\left(f_{k}^{1 i}, k=1, \ldots, 6\right)$ and subsystem 2 by the nodal displacement $\left(u_{k}^{2 i}, k=1, \ldots, 6\right)$. One deduces from the physical interpretation that the interaction modal work between the $\mathrm{p}^{\text {th }}$ mode of subsystem 1 and the $\mathrm{q}^{\text {th }}$ mode of subsystem 2 is expressed by:

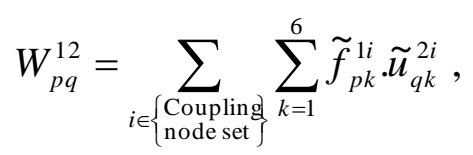

where $\tilde{f}_{p k}^{1 i}$ is the $\mathrm{k}^{\text {th }}$ component force of node $\mathrm{i}$ of the $\mathrm{p}^{\text {th }}$ mode of subsysteml, and $\quad \tilde{u}_{q k}^{2 i}$ is the $\mathrm{k}^{\text {th }}$ component displacement of node $\mathrm{i}$ of the $\mathrm{q}^{\text {th }}$ mode of subsystem 2.

N.B.: All nodal variables must be described in the same global co-ordinate system for the two subsystems.

In conclusion, to calculate the Coupling Loss Factor of complicated system only eigenvalue problem using FEM must be solved for each uncoupled subsystem. The informations, which must be extracted, are the natural angular frequencies, the generalized masses and the mode shapes (nodal forces or nodal displacement) on the coupling boundary (coupling node set). Equation (80) permits to determine the modal interaction works. Then, expression (68) can be applied directly to calculate the CLF between the two subsystems. 


\section{CONCLUSIONS}

One has presented here a technique to calculate SEA Coupling Loss Factors for complicated subsystem modelled with FEM. The technique relies on the basic modal formulation of SEA and the use of a formulation called Dual Modal Formulation. The use of the DMF presents some advantages: (a) the modal equation have no direct coupling between modes of the same subsystem (like SEA assumes); (b) The considered subsystem modes are the physical local modes when weak coupling is respected. Therefore these resonant modes are able to represent the behaviour of the structure in a frequency bandwidth; (c) The natural frequencies which must be considered to determine the CLF are the natural frequencies of the uncoupled (free or blocked) subsystems. (d) There is not the problem of stored energy in the coupling between modes because it is only gyroscopic (no mass and stiffness coupling that store energy are necessary with the present approach); (e) The method is simple to apply because it is possible to use directly the physical interpretation of the interaction modal work.

The final expression of CLF which has be obtained allows us to determine it only from the knowledge of the modes of the uncoupled - subsystems and the modal damping. Finite Element Method can be used to calculate the modal information in the case of complex subsystems that allows one to apply this technique to industrial structures. Contrary to classical SEA matrix inversion technique, the present method does not require solving equations of motion for a lot of excitation. CLF are directly obtained by equation (68) without resolution of equation. This saves an enormous computing time. In addition, one can use different damping loss factor for subsystem without difficulty. In the numerical simulation technique and SEA matrix inversion, one has often the technical difficulty that FEM codes use a global damping loss factor for the considered structure. It is then impossible to use different damping loss factors for substructures.

It can be noticed that it is also possible to establish an ensemble averaged estimate of the CLF by replacing deterministic Intermodal Coupling Factors by ensemble average of the Intermodal Coupling Factors. 


\section{ACKNOWLEDGMENT}

The authors are grateful for the interest and financial support of DGA/DSP-CNRS (Direction des Systèmes de forces et de la Prospective) and CTSN Toulon (Centre Technique des Systèmes Navals), without which this work would not have been done. 


\section{REFERENCES}

[1] R.H. LYON and R.G. DEJONG 1995 Theory and Application of Statistical Energy Analysis, Butterworth-Heineman.

[2] F.F. YAP and J. WOODHOUSE 1996, Journal of Sound and Vibration 197(3), 351-371. Investigation of damping effects on statistical energy analysis of coupled structures.

[3] C. CACCIOLATI and J.L. GUYADER 1994 Phil. Trans. R. Soc. Lond. 346, 465-475. Measurement of SEA coupling loss factors using point mobilities.

[4] G. OREFICE 1997 Ph.D. Thesis, Institut National des Sciences Appliquées de Lyon, France. Description par Mobilités Energétiques des échanges vibratoires dans les systèmes couplés.

[5] K.O. LUNDBERG 1997 Acustica 83, 605-610. A method for estimating SEA loss factors.

[6] F.J. FAHY 1998 Journal of Sound and Vibration 214(2), 261-267. An alternative to the SEA coupling loss factor: rationale and method for experimental determination.

[7] D.A. BIES and S. HAMID 1980 Journal of Sound and Vibration 70(2), 187-204. In situ determination of coupling loss factors by the power injection method.

[8] N. LALOR 1997 The practical implementation of SEA. In: IUTAM Symposium on SEA. ISVR, university of Southampton, UK, 8-11 July 1997 (ed. F.J. Fahy \& W.G. Price, Kluwer Academic Publishers).

[9] J.A. STELL and R.J.M. CRAIK 1994 Journal of Sound and Vibration 178(4), 553-561. Statistical energy analysis of structure-borne sound-transmission by finite-element methods.

[10] C. SIMMONS and C. FREDO 1990, Vibration analysis of coupled stiffened plates using the finite element method. In: internoise'90. International Conference on Noise Control Engineering, Gothenburg, Sweden, August 1990.

[11] C.R. FREDO 1997 Journal of Sound and Vibration 199(4), 645-666. A SEA-like approach for the derivation of energy flow coefficients with a finite element model.

[12] K. SHANKAR and A.J. KEANE 1997 Journal of Sound and Vibration 201(4), 491-513. Vibrational energy flow analysis using a substructure approach: the application of receptance theory to FEA and SEA.

[13] G. MAIDANIK and J. DICKEY 1987 Modal and wave approaches to the Statistical Energy Analysis (SEA). In The winter annual meeting of the American society of mechanical engineers, Massachusetts USA, 13-18 December 1987.

[14] E.E. UNGAR 1966 Fundamentals of Statistical Energy Analysis of Vibrating Systems. U.S. Air Force AFFDL-TR 66-52, April 1966.

[15] F.J. FAHY 1969 Journal of Sound and Vibration 10(3), 490-512. Vibration of containing structures by sound in the contained fluid.

[16] F.J. FAHY 1970 Journal of Sound and Vibration 13(2), 171-194. Response of a cylinder to random sound in the contained fluid.

[17] R.H. LYON and G. MAIDANIK 1962 The Journal of the Acoustical Society of America 34(5), 623-639. Power flow between linearly coupled oscillators.

[18] D. KARNOPP 1966, The Journal of the Acoustical Society of America 40(2),380-384. Coupled vibratory-system analysis, using the dual formulation. 
[19] F.J. FAHY 1994, Phil. Trans. R. Soc. Lond. A346, 431-447. Statistical energy analysis: a critical overview.

[20] L. MAXIT and JL. GUYADER 1999 Statistical modal Energy distribution Analysis (SmEdA). In $6^{\text {th }}$ International Congress on Sound and Vibration, Copenhagen, Denmark, 5-8 July 1999.

[21] S.H. CRANDALL, D.C. KARNOPP, E.F. KURTZ, JR., and D.C. PRIDMORE-BROWN 1968 Dynamics of mechanical and electromechanical systems. Ed. S.H. Crandall, McGraw-Hill Book Company.

[22] R.M. DESEIGNE 1983, Ph.D. Thesis, Institut National des Sciences Appliquées de Lyon, France. Méthodes variationnelles mixtes en élastostatique, application à des structures composites: plaques multicouches et composites plans colles.

[23] P.P. JAMES and F.J. FAHY, An indicator of coupling strength between SEA subsystems. In: IUTAM Symposium on SEA. ISVR, university of Southampton, UK, 8-11 July 1997 (ed. F.J. Fahy \& W.G. Price, Kluwer Academic Publishers).

[24] J.L. GUYADER 1984, Thése de doctorat d'Etat es-science, Institut National des Sciences Appliquées de Lyon and University Claude Bernard Lyion I, France. Méthode d'étude des vibrations des milieux continus imparfaitement caractérisés.

[25] S.H. CRANDALL and R. LOTZ 1971, The Journal of the Acoustical Society of America 49(1),352-356. On the coupling loss factor in statistical energy analysis. 


\section{APPENDIX : NOMENCLATURE}

$a_{p}^{1}(t), a_{q}^{2}(t)$

$b_{p}^{1}(t), b_{q}^{2}(t)$

$c_{p}^{1}(t), c_{q}^{2}(t)$

$E_{1}, E_{2}$

$E_{K}^{1 p}, E_{K}^{2 q}$

$E_{P}^{1 p}, E_{P}^{2 q}$

$E_{p}^{1}, E_{q}^{2}$

$E_{K}^{1}, E_{K}^{2}$

$E_{P}^{1}, E_{P}^{2}$

$f_{k}^{1 i}, f_{k}^{2 i}$

$\tilde{f}_{p k}^{1 i}, \tilde{f}_{q k}^{2 i}$

$F_{1}, F_{2}$

$F_{p}^{1}, F_{q}^{2}$

$\bar{F}_{i}^{c}$

$G_{c}$

$K_{1}, K_{2}$

$K_{p}^{1}, K_{q}^{2}$

$L_{p q}^{1}, L_{p q}^{2}$

$m, n, p, q, r, s$

$M_{1}, M_{2}$

$M_{p}^{1}, M_{q}^{2}$

$n_{j}^{1}, n_{j}^{2}$

$N_{1}, N_{2}$

$S_{i j k l}^{1}, S_{i j k l}^{2}$

$S_{\text {Free }}^{1}, S_{\text {Free }}^{2}$

$S_{\text {Clamped }}^{1}, S_{\text {Clamped }}^{2}$

$S_{\text {Coupling }}^{1}, S_{\text {Coupling }}^{2}$

$t_{0}, t_{1}$

$u_{k}^{1 i}, u_{k}^{2 i}$

$\tilde{u}_{p k}^{1 i}, \tilde{u}_{q k}^{2 i}$

$V^{1}, V^{2}$

$\mathbf{W}_{p q}^{12}$

$W_{i}^{1}, W_{i}^{2}$

$\tilde{W}_{i}^{1 p}, \tilde{W}_{i}^{2 q}$

$\bar{W}_{i}^{c}$

$y_{1}, y_{2}$

$\beta$

$\beta_{p q}^{12}$

$\Delta \omega$ modal amplitude of displacement expansion

modal amplitude of stress expansion

modal amplitude defined by equation (62)

time averaged total energy of oscillator

instantaneous modal kinetic energy

instantaneous modal potential energy

time averaged modal total energy

instantaneous subsystem kinetic energy

instantaneous subsystem potential energy

force nodal variable

force nodal mode shape

external force

generalised 'force'

specified forces on $S_{\text {Coupling }}^{2}$

gyroscopic constant

oscillator's stiffness

modal stiffness

generalised 'forces' and interaction 'forces' with others modes

modal order

oscillator's mass

modal mass

outer normal vector

number of resonant modes in the considered frequency bandwidth

compliance tensor

boundary surface with free displacement

boundary surface with blocked displacement

coupling boundary surface

time

displacement nodal variable

displacement nodal mode shape

volume occupied by subsystem

interaction modal work between mode $\mathrm{p}$ of subsystem 1 and mode $\mathrm{q}$ of subsystem 2

displacement vector

displacement mode shape

specified displacements on $S_{\text {coupling }}^{1}$

oscillator amplitude

proportional constant between $\Pi_{12}$ and $\left(E_{1}-E_{2}\right)$

Intermodal Coupling Factor (ICF) between mode p of subsystem 1 and mode q of subsystem 2

angular frequency bandwidth of interest 
$\Delta_{1}, \Delta_{2}$

$\Delta_{p}^{1}, \Delta_{q}^{2}$

$\varepsilon_{i j}^{1}, \varepsilon_{i j}^{2}$

$\eta_{12}$

$\eta^{1}, \eta^{2}$

$\gamma$

$\gamma_{p q}^{12}$

$\Pi_{1-2}$

$\Pi_{12}$

$\Pi_{p q}^{12}$

$\tilde{\theta}_{y}^{2 q}$

$\omega_{c}$

$\omega_{1}, \omega_{2}$

$\omega_{p}^{1}, \omega_{q}^{2}$

$\Omega_{R}^{1}, \Omega_{R}^{2}$

$\rho^{1}, \rho^{2}$

$\sigma_{i j}^{1}, \sigma_{i j}^{2}$

$\tilde{\sigma}_{i j}^{1 p}, \tilde{\sigma}_{i j}^{2 q}$

$\Sigma_{R}^{1}, \Sigma_{R}^{2}$

$\xi_{1}, \xi_{2}$

$\psi_{R}^{1}, \psi_{R}^{2}$

oscillator's damping bandwidth

modal damping bandwidth

strain tensor

Coupling Loss Factor (CLF)

Damping Loss Factor (DLF)

gyroscopic coupling coefficient between two oscillators

gyroscopic modal coupling coefficient between mode p of subsystem 1 and

mode $\mathrm{q}$ of subsystem 2

time averaged power flow exchanged by two subsystems

time averaged power flow from oscillator 1 to oscillator 2

time averaged power flow from mode $\mathrm{p}$ of subsystem 1 to mode $\mathrm{q}$ of subsystem 2

angular rotation mode shape of beam 2

central angular radian frequency of the frequency bandwidth $\Delta \omega$

oscillator's natural angular frequency

mode's natural angular frequency

displacement admissible space

mass density

stress tensor

stress mode shape

stress admissible space

time averaged total energy of subsystem

Reissner functional 


\section{FIGURE CAPTIONS}

Figure 1: Two oscillators coupled by gyroscopic element.

Figure 2: $\quad$ Illustration of coupling of $\mathrm{N}_{1}$ modes of subsystem 1 with $\mathrm{N}_{2}$ modes of subsystem 2 .

Figure 3: Representation of the coupling of the two elastic continuum systems.

Figure 4: Illustration of the fictive separation of subsystems.

Figure 5: Subsystem definition (a) Uncoupled - blocked subsystem $1 \quad$ (b) uncoupled - free subsystem 2 .

Figure 6: Illustration of the oscillator associated to the $\mathrm{p}^{\text {th }}$ blocked modes. 


\section{FOOTNOTE:}

Foot note 1: It may be noted that several typing errors and one error of sign are present in the equations of this paper. 


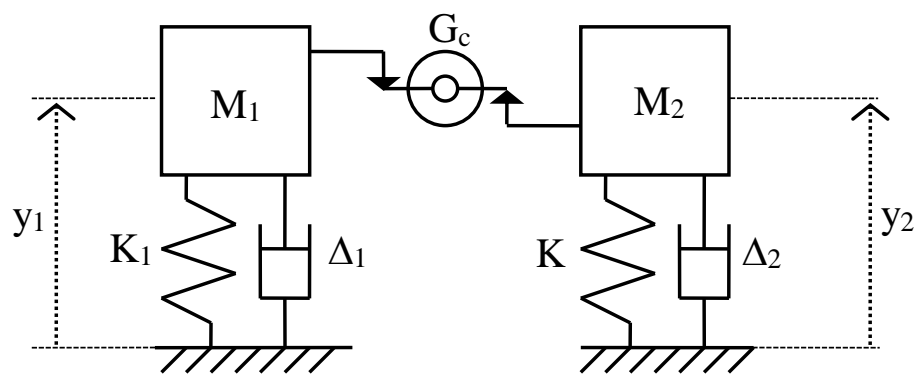


FIGURE 2

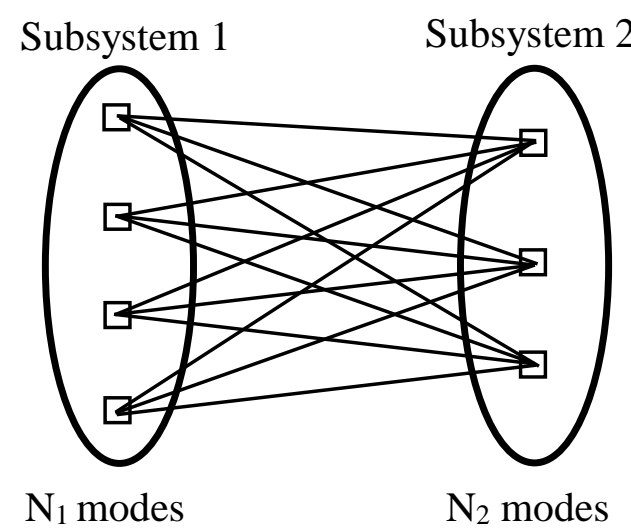


FIGURE 3

L.MAXIT, J.L. GUYADER

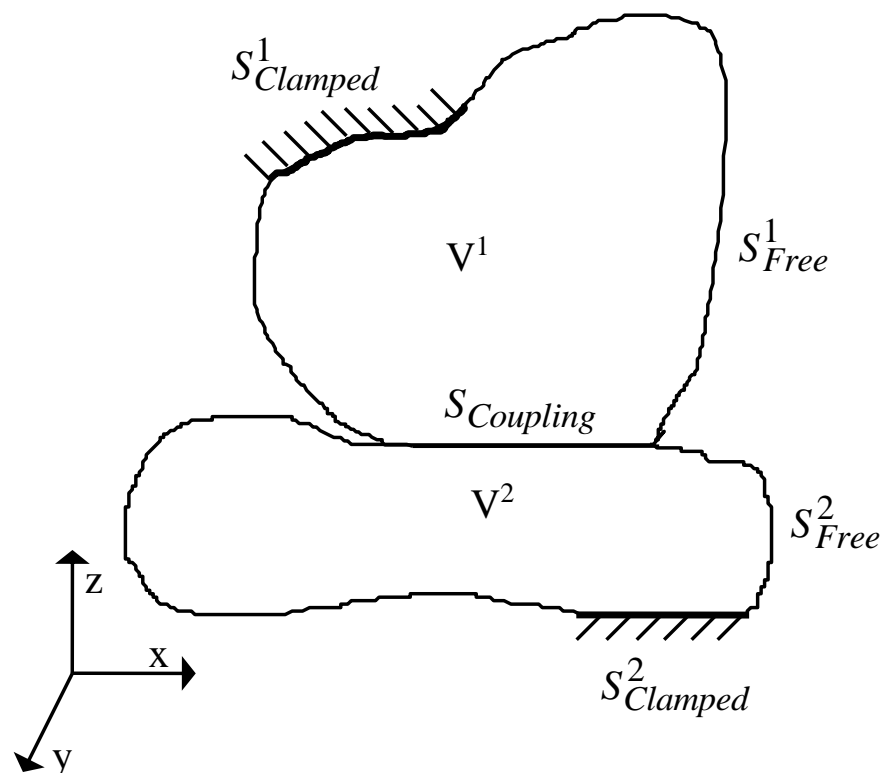


FIGURE 4

L.MAXIT, J.L. GUYADER

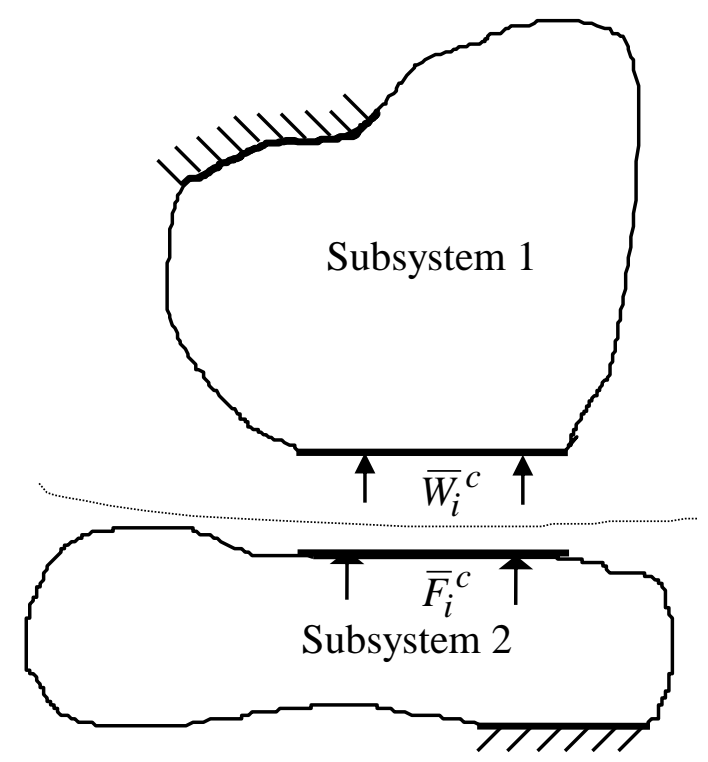


FIGURE 5

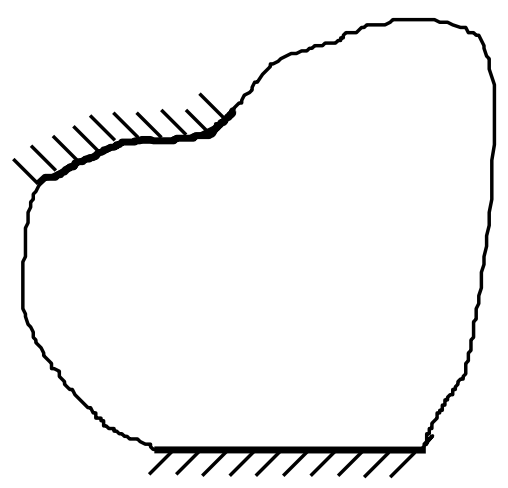

L.MAXIT, J.L. GUYADER

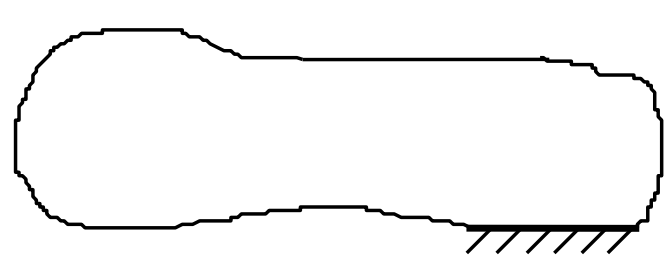


FIGURE 6

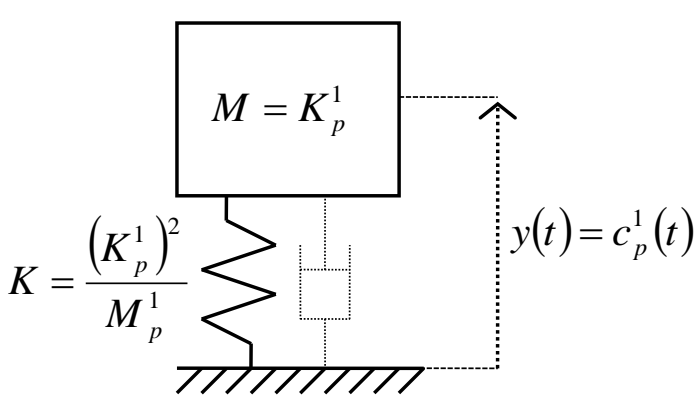

